\title{
Does Dimercaptosuccinic acid (DMSA) scan yield additional information following non-structural renal ultrasound scans findings?
}

K Maduemem, Y Diaz Rodriguez, B Fraser

Department of Paediatrics, Cork University Hospital, Ireland

\section{Background and Aims}

- Urinary tract infection (UTI) is one of the common presentations in acute Paediatrics

- Radiological investigations are essential to rule out structural renal tract anomalies and consequently, reduce morbidity

- In Ireland, these investigations are performed based on NICE guidance until recently

- Irish guidelines have proposed performing a repeat renal ultrasound scan (RUSS) in place of DMSA scan following nonstructural RUSS findings

- We aimed to evaluate additional information of DMSA scans following RUSS in children with UTI

\section{Methods}

- This was a retrospective study of children admitted with documented culture positive UTI over a 5-year-period in a regional hospital

- Investigations performed based on NICE guidance were evaluated

- Radiological findings of DMSA scans and RUSS were compared using chi-squared test

\section{Results}

\begin{tabular}{l|r} 
Total number of children & 470 \\
\hline Male sex (\%) & $211(44.9)$ \\
\hline Median age at presentation (range) years & $0.2(0.02-15.95)$ \\
\hline Median length of stay (range) days & $3(0.6-22)$
\end{tabular}

\begin{tabular}{l|r|rr} 
& Normal RUSS & Abnormal RUSS \\
\hline Normal DMSA & 71 & 30 \\
\hline Abnormal DMSA & 7 & 33
\end{tabular}

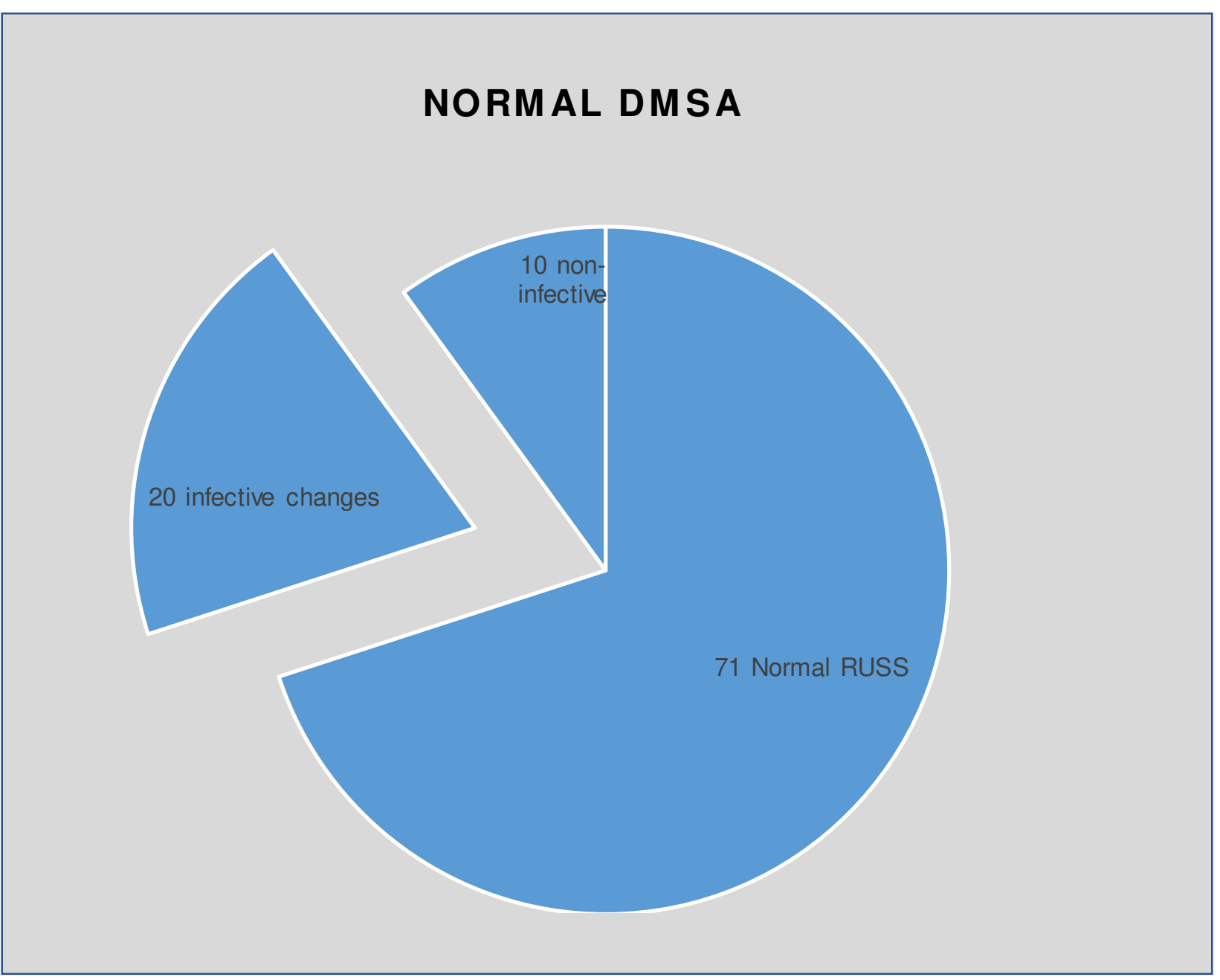

\section{Results}

- RUSS was done on 394 children with 154 reported as abnormal. 37\% (58/154) reported as infective changes without gross structural anomalies

- Infective changes on RUSS were associated with normal DMSA scan 4 to 6 months later $(p<0.05)$

- The sensitivity and specificity of RUSS in detecting vesico-ureteric reflux (VUR) were 0.45 (95\% Cl: $0.35-0.56)$ and 0.71 (95\% Cl: 0.57-0.82) respectively

\section{Conclusion}

This study demonstrated no significant benefit of DMSA scans following non-structural RUSS findings

MCUG remains invaluable in VUR diagnosis

$>$ A repeat RUSS following non-structural RUSS findings would minimize the cost and radiation risks of DMSA scans 